\title{
Latent orientation in the skin layer of electrospun isotactic polystyrene ultrafine fibers
}

\author{
$\operatorname{AUTHOR}(\mathrm{S})$ : \\ Tosaka, Masatoshi; Yamaguchi, Katsuya; Tsuji, \\ Masaki
}

\section{CITATION:}

Tosaka, Masatoshi ...[et al]. Latent orientation in the skin layer of electrospun isotactic polystyrene ultrafine fibers. Polymer 2010, 51(2): 547-553

\section{ISSUE DATE:}

2010-01-21

URL:

http://hdl.handle.net/2433/96289

\section{RIGHT:}

c 2009 Elsevier Ltd. All rights reserved.; This is not the published version. Please cite only the published version.; この論文は出版社版でありませ ん。引用の際には出版社版をご確認ご利用ください。 


\title{
Latent orientation in the skin layer of electrospun isotactic polystyrene ultrafine fibers
}

\author{
Masatoshi Tosaka*, Katsuya Yamaguchi, Masaki Tsuji \\ Institute for Chemical Research, Kyoto University \\ Uji, Kyoto 611-0011, Japan
}

* Corresponding author

Tel: +81-774-38-3062, Fax: +81-774-38-3069, E-mail: tosaka@scl.kyoto-u.ac.jp

\begin{abstract}
Isotactic polystyrene was electrospun into ultrafine fibers from the chloroform or tetrahydrofuran solutions. Tubular or ribbon-like fibers with many small pores were obtained from the chloroform solution. Densely stacked lamellae were formed by annealing them. On the other hand, fibers with many small dents on the skin layer were obtained from the tetrahydrofuran solution. The skin layer changed into the densely stacked lamellae by annealing, while the interior was not crystallized under the utilized annealing condition. These results suggested that the skin layer of the as-spun fiber should contain a finite amount of highly oriented molecular chains, while the interior may be unoriented. Despite the annealing, the orientation of the chains in the skin layer has been preserved and worked as the nuclei for the densely stacked lamellae.
\end{abstract}

\section{KEY WORDS}

electrospinning, concentration gradient, stacked lamellar structure 


\section{Introduction}

Recently, electrospinning is receiving great attention as a technique to produce various ultrafine polymeric fibers that are difficult to fabricate by other conventional methods. The diameters of the fibers produced by electrospinning are sometimes smaller than one micrometer. Remarkable characteristics of the ultrafine fibers are the high ratio of surface area to mass, the unique hydrodynamic effects, and the optical properties which result from the smaller diameter than wavelength of visible light. These characteristics will lead to development of novel materials with unique functionality in the field of biology, medical science and engineering. Thus electrospinning is expected to be one of the key technologies to produce such novel materials. Over 2000 publications including journal papers and patents on electrospinning have been published in the last decade [1]. The topics of these publications are mostly the electrospinning conditions, characterizations of electrospun fibers and fundamentals of electrospinning.

While the formation mechanism of the ultrafine jet of a polymer solution during electrospinning process has been studied in detail [2], studies on structures of electrospun ultrafine fibers have been limited mostly to their exterior view. To our knowledge, internal fine structure of a single electrospun fiber is scarcely reported. One of the reasons for the absence of such studies lies in experimental difficulties. In this case, the "internal structure" is examined as distribution of molecular orientation or density fluctuation in the ultrafine fibers. When the polymer chains are in the amorphous state, these characteristics in the ultrafine fibers are extremely difficult to detect; at most, average degree of molecular orientation or density fluctuation may be examined by, e.g., X-ray diffraction/scattering, supposing sample amounts are enough for such examinations. Analysis of distribution and orientation of crystals is, on the other hand, possible even in the ultrafine fibers by dark-field (DF) imaging of transmission electron microscopy (TEM). We have indeed observed distribution of specific crystalline domains in single lamellar crystals in molecular resolution [3-5]. If the ultrafine fibers crystallize reflecting the orientation of the molecular chains in the as-electrospun fibers, we will be able to discuss the internal fine structure and its formation mechanism during spinning in the electrospun fibers. 
The crystalline polymer species suitable for this purpose must be, of course, the one that can form the ultrafine fibers by electrospinning. In order to regard the samples as a representative model of electrospun fibers, flexible-chain polymers are preferable. However, highly crystalline polymers may be unsuitable, because rapid crystallization during the spinning process can perturb the distribution of molecular orientation in the spinning jet. On the basis of the above-mentioned consideration, we have selected isotactic polystyrene (i-PS) as the polymer to be studied. Because there have been some reports that atactic polystyrene (a-PS) was electrospun into the ultrafine fibers [2,6-8], iPS was also expected to be electrospun. (To our knowledge, this paper may be the first report on electrospinning of i-PS.) This polymer has relatively flexible molecular chains. Additionally, crystallization rate of i-PS is known to be moderate [9].

In this paper, electrospun i-PS ultrafine fibers are studied by TEM. Some interesting features of the i-PS fibers will be reported and the internal fine structures are discussed on the basis of distribution of the crystallites.

\section{Experimental section}

The i-PS used in this study ( $\mathrm{Mw}=400,000 、 90 \%$ isotacticity) was purchased from Scientific Polymer Products, Inc. Tetrahydrofuran (THF) and chloroform were purchased from Wako Chemical, Inc. and used as the solvents without further purification. For electrospinning, 0.5 or $1.0 \mathrm{wt} \%$ solutions of i-PS were prepared using each solvent. The ultrafine fibers were electrospun using a commercial instrument (Fuence Co. Ltd., esprayer ES-1000) equipped with a hand-made "parallel-electrode collector" containing a gap in its middle $[10,11]$, which was made on the basis of the reports by $\mathrm{Li}$ and others $[6,12]$. This special collector serves to align the electrospun fibers in one direction for the easier analysis of molecular orientation. (Fairly parallel fibers are deposited on the collector, bridging the gap between two parallel electrodestrips in a fashion perpendicular to the edge of the strip.) The distance between the nozzle and the collector was $75 \mathrm{~mm}$. The width of the gap in the collector was $3 \mathrm{~mm}$. The parameters for electrospinning were determined according to preliminary studies to obtain ultrafine fibers which have diameters as thin as $250 \mathrm{~nm}$. The chamber of the electrospinning instrument was conditioned at $25{ }^{\circ} \mathrm{C}$, while relative humidity in the laboratory room varied between $30 \%$ and $60 \%$. The feeding rate of the solution was 20 $\mu \mathrm{l} / \mathrm{min}$. The applied voltage for electrospinning was 15 or $20 \mathrm{kV}$. The combination of 
the solution concentration and the applied voltage for electrospinning will be indicated as occasion arises (e.g., in the corresponding figure caption).

The electrospun fibers were picked up on a cupper grid having a carbon supporting-film for TEM. Some of the cupper grids with the electrospun fibers were annealed in air using a hot stage. The annealing conditions were also determined according to preliminary experiments. The samples were heated up to a prefixed annealing temperature at a rate of $10^{\circ} \mathrm{C} / \mathrm{min}$. The temperature and length of annealing will be indicated in the corresponding figure caption.

The specimens thus prepared for TEM were observed by a transmission electron microscope (JEOL Ltd., JEM-200CS) operated at $200 \mathrm{kV}$. For the stereoscopic viewing, two images of a sample were taken; one of the images was observed in the direction tilted by $5^{\circ}$ from the other by rotating the sample around an axis perpendicular to the viewing direction, namely to the incident beam direction. These two images were put on a plane as they are shifted in the direction perpendicular to the tilting axis. By viewing the slightly different two images (so-called the stereogram) using the right and left eyes separately, the human brain can obtain the perception of three-dimensional shape of the sample. For subtraction of background to highlight the stacked-lamellar structure, the TEM image was processed by the "Image J" software (http://rsb.info.nih.gov/ij/).

\section{Results and Discussion}

Though the diameter was fluctuated in each case, the two types of solvents for electrospinning have led to distinct features of the i-PS ultrafine fibers.

Electrospun fibers from the chloroform solution.

Fig. 1 shows an example of the as-spun ultrafine fiber from the $0.5 \mathrm{wt} \%$ chloroform solution at $15 \mathrm{kV}$. In the bright-field (BF) TEM image (Fig. 1a), we can notice many bright spots in the fiber. The width of the fiber in Fig. 1a exceeds $1 \mu \mathrm{m}$. If the fiber has a cylindrical shape filled with the polymer molecules, the TEM image must be much darker, in particular around the central part of the fiber, because the electron beam cannot pass through such a thick specimen. These features as recognized in Fig. 1a were observed in many other as-spun fibers from the chloroform solution in our experiment. In order to understand the morphological features of the ultrafine fibers, we observed them by the stereoscopic viewing. An example of the stereogram used for this investigation is shown in Fig. 1c. By stereoscopic viewing of Fig. 1c, it is known that 
this fiber has tubular shape and the right side creases toward the inside. On the basis of such stereograms, we found that most of the as-spun fibers from chloroform solution under this experimental condition have tubular or ribbon-like shapes with many small pores. For the convenience of readers who cannot perform the stereoscopic viewing, schematic drawing of the tubular and ribbon-like fibers are presented in Fig. 2.

Formation of the tubular or ribbon-like fibers as a result of electrospinning has been explained by Guenthner et al. on the basis of the numerical simulation of the development of concentration gradient in the solution jet [13]. Because the evaporation of chloroform is very fast, the surface of the solution jet can have considerably solidified while the interior can have contained a lot of solvent. Then evaporation of the solvent from the interior can have generated vacancies, leading to the tubular shape. The ribbon-like shape may have formed by the collapse of the tube due to the solvent evaporation.

On the other hand, the formation mechanism of the pores is currently unclear. Because the formation of such pores is not reported for electrospun a-PS ultrafine fibers, we suspect that crystallinity of i-PS is related to this phenomenon. As shown in the selected-area electron diffraction (SAED) pattern (Fig. 1b), the as-spun fibers contain a small amount of oriented crystals. If there was distribution of crystallites in the skin layer of the solution jet, degree of solidification may have been different from place to place. Then the poorly solidified regions could have become the pores.

In order to access the distribution of molecular orientation in the as-spun i-PS fibers, we observed them by DF imaging. Unfortunately, we could not obtain meaningful DF images from these samples. The crystallites in the as-spun i-PS fiber may have been so small and imperfect that they cannot produce perceivable contrast in the DF images. For the enhancement of the crystalline contrast in DF images, the size and perfection of the i-PS crystals must be increased. We therefore annealed the electrospun i-PS fibers.

Fig. 3 shows an example of the annealed electrospun i-PS fiber from the chloroform solution. As the BF image (Fig. 3a) shows, we have found no pores in the annealed fibers. In the case of the as-spun specimens, fibers bridging the mesh of the TEM copper-grid were sometimes observed even at locations where the supporting carbon films were broken off. On the other hand, in the case of the annealed specimens, 
fibers such as in Fig. 3 were found only on the supporting film. Accordingly, we concluded that the i-PS fibers were melted during the annealing and the pores evanesced due to their liquescence on the supporting film; fibers without the supporting film may have flowed away. Usually, melting of slightly oriented polymer will lead to relaxation of molecular chains. However, the electrospun ultrafine fibers of i-PS did not follow this expectation. The SAED pattern (Fig. 3b) indicates that the crystallinity of the sample is very high with high degree of molecular orientation. Detailed inspection of the BF image (Fig. 3a) also revealed the formation of highly-oriented and densely stacked crystalline lamellae, as shown in Fig. 3c. The long period of the stackedlamellar structure, which was measured as the average value for several parts in Fig. 3c, was $12 \mathrm{~nm}$.

Fig. 4 shows the DF images of the annealed i-PS fiber in Fig. 3. In this case, crystallites meeting the diffraction condition distributed in the sample almost uniformly. Traces of the pores were not recognized in the DF images, which also indicates that the fiber was melted. The possible mechanism for the formation of the highly-oriented fiber from the melted state during the annealing will be discussed later.

\section{Electrospun fibers from the THF solution}

Fig. 5 shows an example of the as-spun i-PS ultrafine fiber from $1.0 \mathrm{wt} \% \mathrm{THF}$ solution at $20 \mathrm{kV}$. Compared to the fibers from the chloroform solution (Fig. 1a), the image of the fiber in Fig. 5a is much darker despite the smaller width. Accordingly, we can understand that the interior of this fiber is filled with the polymer molecules. The shapes of the electrospun fibers from the THF solution were not cylindrical. For example, the bright band in the fiber running parallel to the fiber axis in Fig. 5a indicates that this part is thinner than the surrounding parts. At the same time, these fibers had finer textures. Fig. 5c shows the enlarged view of this fiber. On the surface (especially, on the both sides) of the fiber, we can recognize bright and dark parts. The bright contrast indicates the less presence of polymer molecules, that is to say, these parts correspond to small dents. Thus we can understand that these fibers have many small dents on the surface.

The shape of the electrospun fibers from the THF solution and its possible formation process are illustrated in Fig. 6. Evaporation of THF is not as fast as chloroform, and accordingly, the concentration gradient in the solution jet [13] can 
become moderate. When the surface of the solution jet was solidified, concentration of the interior might be so high that the evaporation of the solvent from the interior could induce contraction of the jet rather than the formation of the tubular shape. As a result, the fibers were wrinkled to have irregular cross-sections. Many small pores on the skin layer may have been formed by the same mechanism as the case of the electrospinning from the chloroform solution.

In the SAED pattern in Fig. 5b, we can recognize neither trace of orientation nor crystalline reflections, though we sometimes observed the oriented crystalline reflections like the ones in Fig. 1b in other specimens from the THF solution. In any cases, again, we could not obtain meaningful DF images of the as-spun i-PS fibers from the THF solution. Accordingly, these samples were also annealed for the DF imaging.

Fig. 7 shows an example of the electrospun i-PS fiber from the THF solution after annealing. In this case, again, the SAED pattern (Fig. 7b) indicates the high degree of crystallinity and molecular orientation of the annealed fibers. The small pores on the surface layer have disappeared by the annealing (Fig. 7a). Alternatively, the stackedlamellar structure was developed, as shown in the background-subtracted image (Fig. 7c). In Fig. 7c, dark striations running in the horizontal direction, in particular those observed more clearly in the skin region along the right-hand edge of the fine fiber, are able to be attributed each to a crystalline edge-on lamella. It is interesting to notice that the structure consisting of highly oriented stacked lamellae was formed from the hardly oriented as-spun fibers by the annealing.

DF images of the annealed i-PS fiber are shown in Fig. 8. Crystallites meeting the diffraction condition for the 102 reflection are distributed almost uniformly (Fig. 8a). On the other hand, crystallites meeting the diffraction condition for the 220 reflection are localized on both sides of the fiber in Fig. 8b. These features in the DF images were frequently found also for other annealed specimens. The DF images in Fig. 8 suggest specific orientation and distribution of the crystallites, which is explained using Fig. 9. In the upper part of Fig. 9, the direction of the incident electron beam is vertical, and accordingly, the crystallites (hexagons) meeting the diffraction condition have the (220) plane (stripes) which is almost parallel to the vertical direction. If the randomlydistributed crystallites were in the fiber orientation, that is to say, with the uniaxial alignment of the $c$-axis and the statistical alignments of $a$ - and $b$-axes around the $c$-axis, 
the more crystallites meeting the diffraction condition (the hexagons with the vertical stripes) would be observed near the center of the fiber because of the larger thickness. Such a hypothetical situation is illustrated in Fig. 9a. On the other hand, the localized detection of the crystallites in the experiments (Fig. 8b) suggests that the crystallites (hexagons) on the both sides of the fiber should have (220) plane (stripes) preferentially oriented in the vertical direction in the upper part of Fig. 9; in the center part of the fiber, such crystallites should be scarce. Assuming the cylindrical symmetry of the fiber, we arrive at the orientation and distribution of the crystallites illustrated in Fig. 9b to explain the features in Fig. 8b. The crystallites should be mostly located near the surface of the fiber, and furthermore, the vector normal to the (220) plane preferentially oriented in the radial direction of the fiber. That is to say, the crystallites may be biaxially oriented in the skin layer.

\section{Possible mechanism for the orientation of the fibers by annealing}

In the above-described observation of the annealed ultrafine fibers, we can deduce the following two phenomena. One is that most of the molecular chains in the as-spun fiber have been experienced the melted state during the annealing process. Otherwise, the reorganization of the surface structure that obliterated the pores is difficult to explain. The other is the existence of oriented molecular chains in the as-spun fibers, which preserve the orientation during the above-mentioned melted state and work as nuclei for the formation of the stacked-lamellar structure. The similar situation is observed in the case of strain-induced crystallization of cross-linked natural rubber. Like the SAED pattern in Fig. 1b, highly stretched samples of cross-linked natural rubber exhibit the highly oriented crystalline reflections and almost isotropic amorphous halo simultaneously in the X-ray diffraction pattern [14-19]. The orientation of molecular chains in natural rubber is preserved because of the network structure whose molecular weight is regarded as infinite; the isotropic halo comes from the liquid-like component [19]. In this sense, the molecular chains which preserve the orientation in the electrospun i-PS fiber would have had very high molecular weight.

On the basis of these deductions, the possible mechanism for the orientation of the electrospun i-PS ultrafine fibers is explained as follows. During the electrospinning process, the skin layer of the solution jet should have had relatively high viscosity because of the evaporation of the solvent [13]. As the jet is elongated due to the 
electrostatic repulsion between electric charges of same sign on its surface [2,20], only the molecular chains in the viscous skin layer can have oriented along the spin line. The still solution-rich interior of the jet seems not to be so viscous, and accordingly, such orientation cannot be developed there. Because the jet contained considerable amount of solvent at this moment, the chains in the skin layer with low molecular weight might be also relaxed before the complete solidification. Only the chains with high molecular weight can have preserved the orientation until the complete solidification. However, the amount of the latter might be limited, and accordingly, the diffraction from the entire fiber might have shown the scarce orientation.

By heating the amorphous molecular chains above the glass transition temperature, they turned into the melted state. Because the annealing temperature was below the melting temperature, however, some of the oriented chains with high molecular weight might be stabilized as crystal nuclei. In the skin layer, oriented lamellar crystals may have subsequently grown from the oriented nuclei, in a similar manner of so-called shish-kebab formation.

Considering the formation mechanism of the tubular electrospun fiber, the entire body of the sample from the chloroform solution may be regarded as the skin layer. Because the collapsed tube of the skin layer can have crystallized uniformly, the crystallites in the DF image in Fig. 4 are not localized. On the other hand, in the electrospun fiber from the THF solution, the stacked-lamellar structure seems to have grown from the skin layer; the interior of the fiber seems still not to have crystallized. The reason for the radial crystalline orientation of the surface layer (Fig. 9b) is still unclear for this sample. We guess that the confinement of the oriented molecular chains near the skin layer may be related to the mechanism of the radial orientation.

\section{Conclusion}

Ultrafine fibers of i-PS were successfully obtained from each of the chloroform and the THF solutions by electrospinning. From the chloroform solution, tubular or ribbon-like fibers with many small pores were obtained. By annealing the fiber below the melting temperature, densely stacked lamellar crystals were formed. Also from the THF solution, fibers with many small pores on the skin layer were obtained. In this case, however, the fibers were not tubular. By annealing, the latter fiber also generated the densely stacked lamellar crystals on the skin layer. Formation of the highly oriented 
lamellar crystals by annealing strongly suggested that the skin layer of the electrospun fiber should contain a finite amount of highly oriented molecular chains, while the interior may be completely unoriented.

\section{ACKNOWLEDGMENT}

This study was partly supported by a Grant-in Aid for Scientific Research (C), No. 20550187 (to M. Tosaka) and No. 19550207 (to M. Tsuji) from Japan Society for the Promotion of Science.

\section{REFERENCES}

1. Zhou F-L, Gong R-H. Polym Int 2008; 57: 837-845.

2. $\quad$ Reneker DH, Yarin A L. Polymer 2008; 49: 2387-2425.

3. Tosaka M, Hamada N, Tsuji M, Kohjiya S, Ogawa T, Isoda S, Kobayashi T. Macromolecules 1997; 30: 4132-4136.

4. Tosaka M, Tsuji M, Kohjiya S, Cartier L, Lotz B. Macromolecules 1999; 32: 4905-4911.

5. Tosaka M, Kamijo T, Tsuji M, Kohjiya S, Ogawa T, Isoda S, Kobayashi T. Macromolecules 2000; 33: 9666-9672.

6. $\quad$ Li D, Wang Y, Xia Y. Nano Lett 2003; 3: 11167-1171.

7. $\quad$ Eda G, Liu J, Shivkumar S. Eur Polym J 2007; 43: 1154-1167.

8. $\quad$ Uyar T, Besenbacher F. Polymer 2008; 49: 5336-5343.

9. Hoffman JD, Davis GT, Lauritzen Jr IJ. In: Hannay NB, editors. Treatise on Solid State Chemistry, Vol. 3. New York: Plenum Press, 1976. pp. 497-614.

10. Nakayama A, Kawahara Y, Hayakawa Y, Takahashi R, Yoshioka T, Tsuji M. Sen'i Gakkaishi 2007; 63: 230-234.

11. Nakayama A, Takahashi R, Hamano T, Yoshioka T, Tsuji M. Sen'i Gakkaishi 2008; 64: 32-35.

12. Li D, Wang Y, Xia Y. Adv Mater 2004; 16: 361-366.

13. Guenthner A J, Khombhongse S, Liu W, Dayal P, Reneker HD, Kyu T. Macromol Theory Simul 2006; 15: 87-93.

14. Murakami S, Senoo K, Toki S, Kohjiya S. Polymer 2002; 43: 2117-2120.

15. Toki S, Sics I, Ran S, Liu L, Hsiao BS, Murakami S, Senoo K, Kohjiya S. Macromolecules 2002; 35: 6578-6584. 
16. Tosaka M, Kohjiya S, Murakami S, Poompradub S, Ikeda Y, Toki S, Sics I, Hsiao B S. Rubber Chem Technol 2004; 77: 711-723.

17. Tosaka M, Murakami S, Poompradub S, Kohjiya S, Ikeda Y, Toki S, Sics I, Hsiao BS. Macromolecules 2004; 37: 3299-3309.

18. Tosaka M. Polymer J 2007; 39: 1207-1220.

19. Tosaka M. Macromolecules 2009; 42: 6166-6174.

20. Ramakrishna S, Fujihara K, Teo W-E, Lim T-C, Ma Z. An Introduction to Electrospinning and Nanofibers. Singapore: World Scientific, 2005. 


\section{FIGURES}

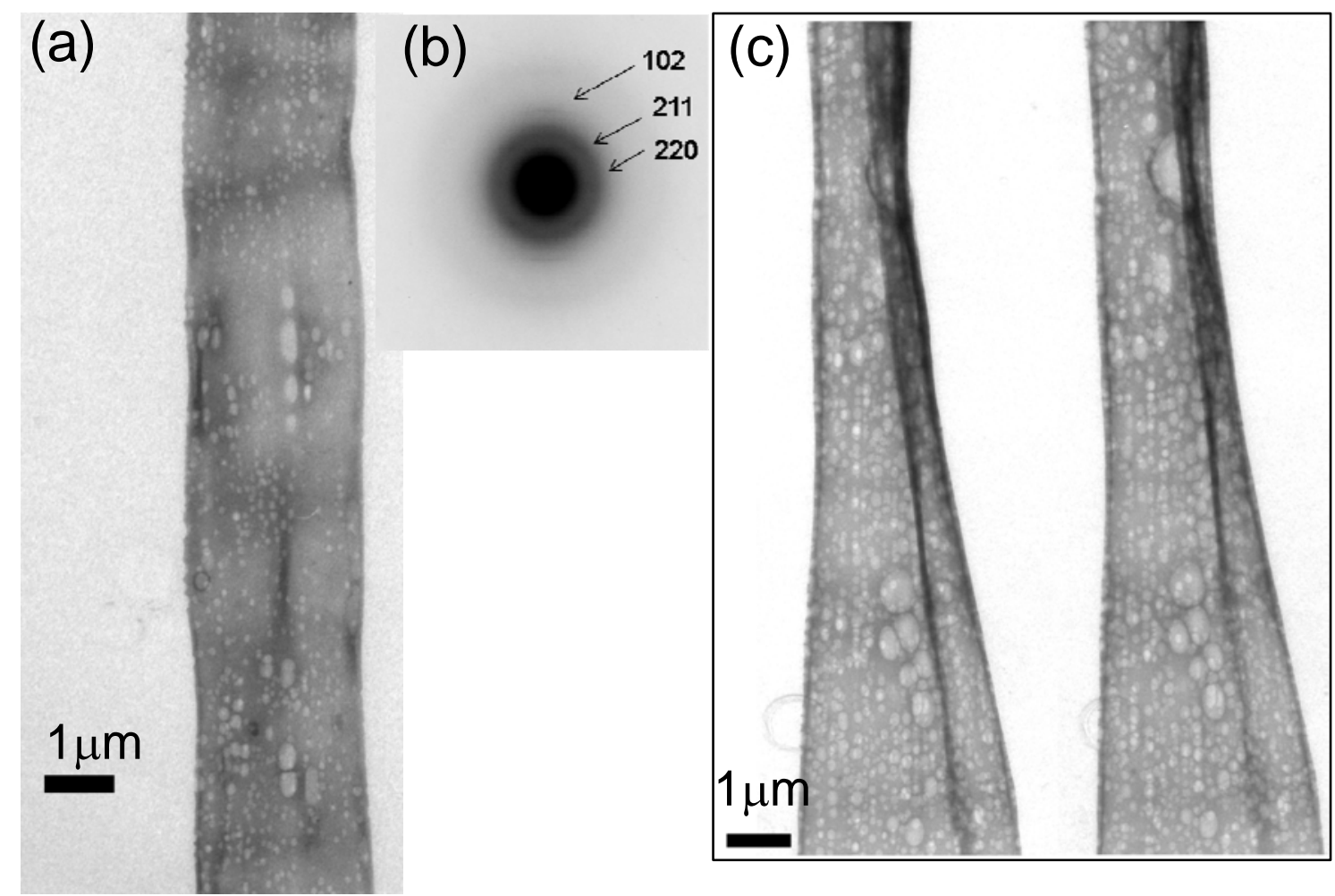

Fig. 1 As-spun ultrafine fiber of i-PS electrospun from the $0.5 \mathrm{wt} \%$ chloroform solution. The voltage for electrospinning was $15 \mathrm{kV}$. (a) Typical BF image of the sample. Many small pores are observed as bright spots. (b) SAED pattern of the sample in part a. Oriented crystalline reflections are slightly seen. (c) Stereogram of another specimen prepared under the same experimental condition. 


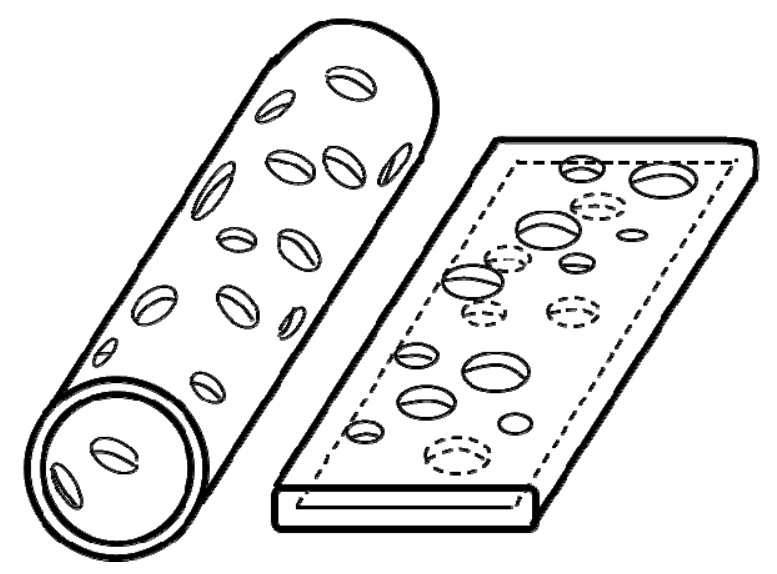

Fig. 2 Schematic drawing of the tubular (left) or the ribbon-like (right) fibers with many small pores. 

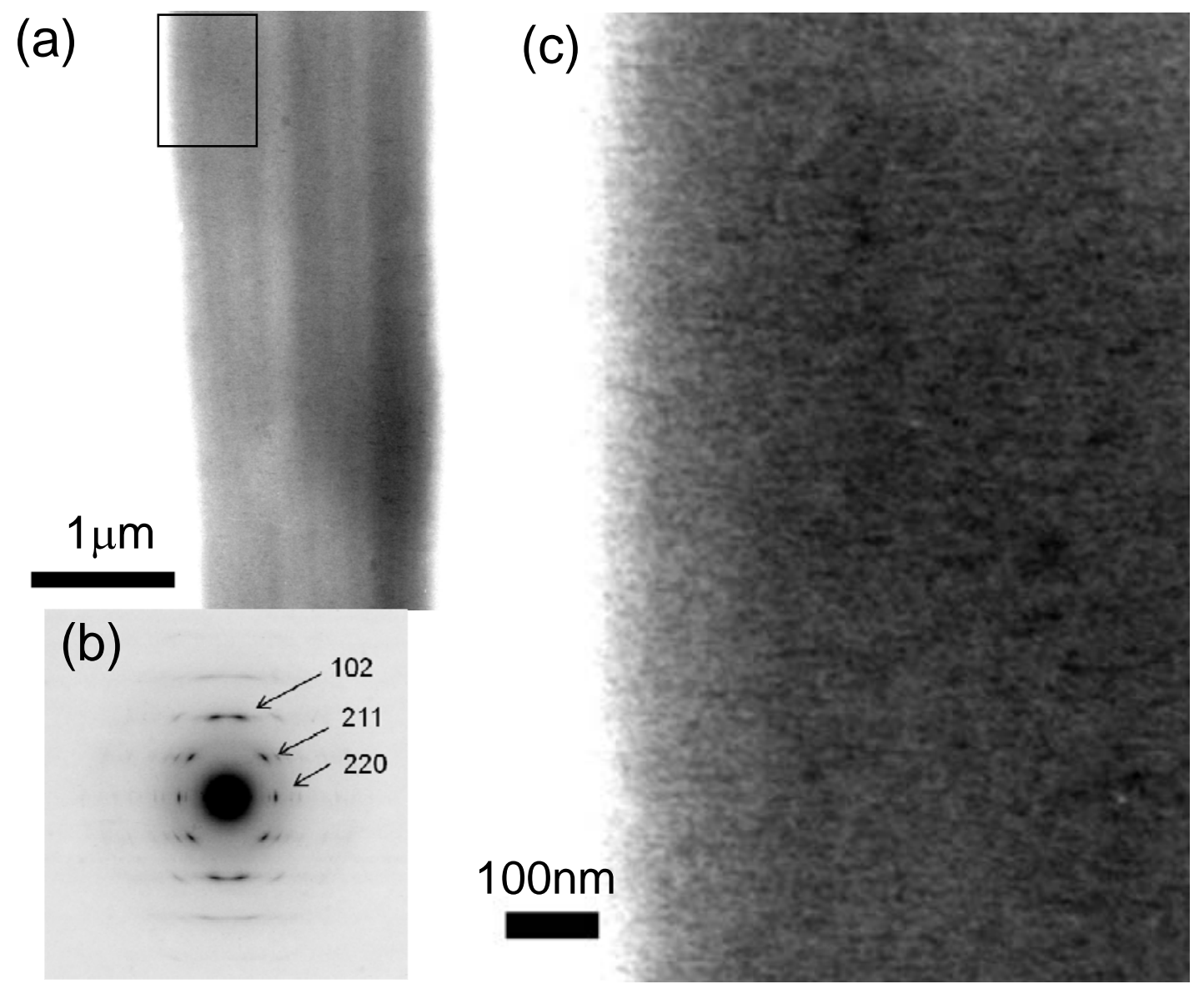

\section{$100 \mathrm{~nm}$}

Fig. 3 Annealed ultrafine fiber of i-PS electrospun from the $0.5 \mathrm{wt} \%$ chloroform solution. The voltage for electrospinning was $15 \mathrm{kV}$. Temperature and the length of annealing were $220^{\circ} \mathrm{C}$ and $18 \mathrm{~h}$. (a) BF image. No pores are observed in this picture. (b) SAED pattern. Strong reflections of oriented crystals are observed. (c) Enlarged view of part a indicated by the rectangle. Horizontal striations corresponding the stacked lamellae are recognized. 


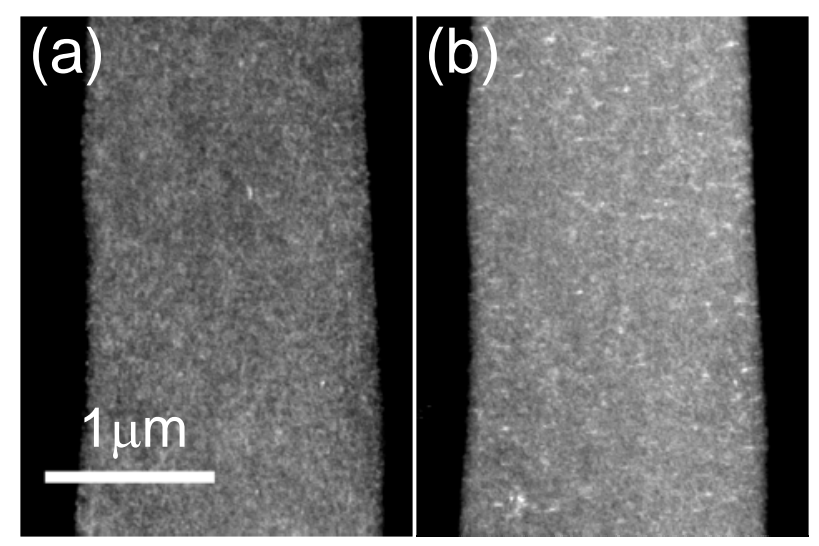

Fig. 4 DF images of the annealed ultrafine fiber of i-PS electrospun from the $0.5 \mathrm{wt} \%$ chloroform solution. The voltage for electrospinning was $15 \mathrm{kV}$. Temperature and the length of annealing were $220^{\circ} \mathrm{C}$ and $18 \mathrm{~h}$. Reflections for the DF imaging were 102 (a) and 220 (b). 


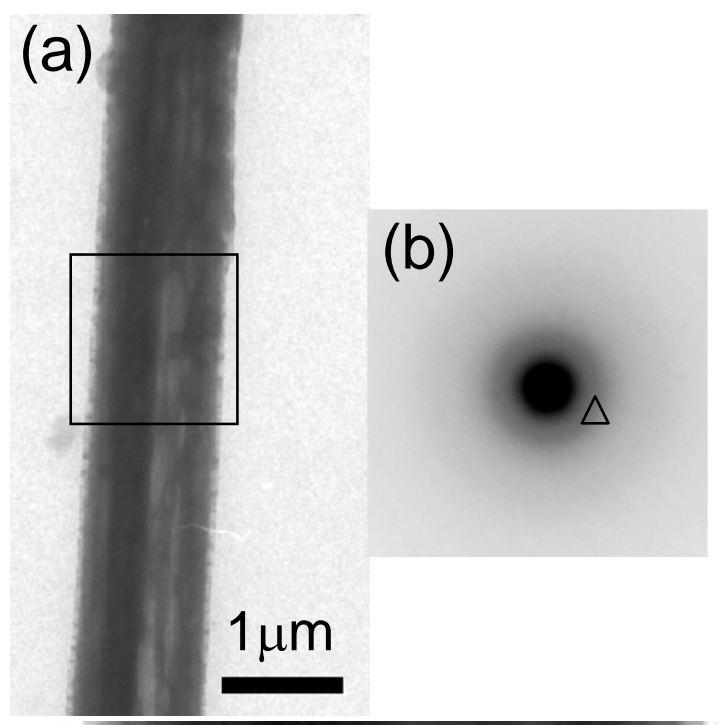

\section{(c)}

\section{$500 \mathrm{~nm}$}

Fig. 5 As-spun ultrafine fiber of i-PS electrospun from the $1.0 \mathrm{wt} \%$ THF solution. The voltage for elecrrospinning was $20 \mathrm{kV}$. (a) BF image. The bright part running lengthwise is thinner than the surrounding parts. (b) SAED pattern. Trace of orientation or crystalline reflections are hardly seen. The location corresponding to the 220 reflection is indicated by the arrowhead. (c) Enlarged view of part a indicated by the rectangle. 


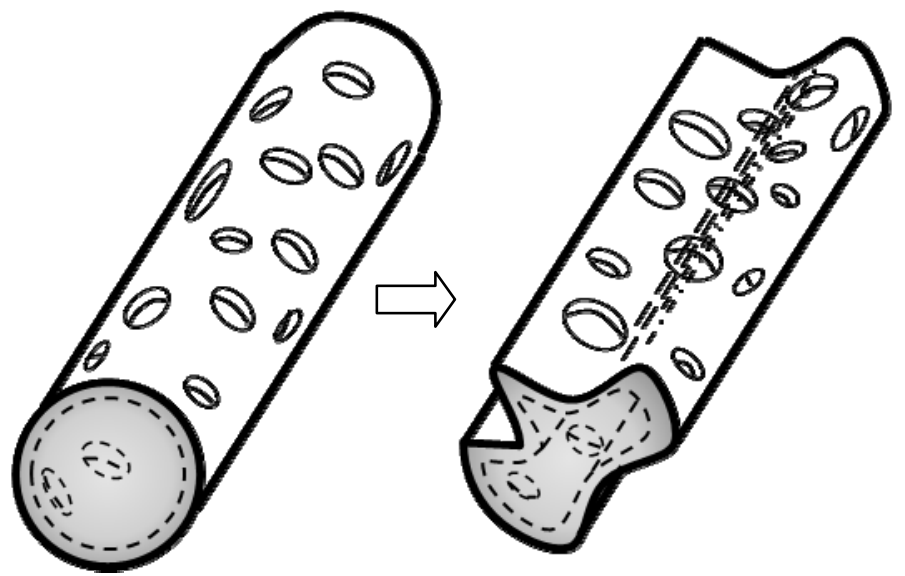

Fig. 6 Schematic drawing of a possible formation process of the ultrafine fiber from the THF solution. The skin layer of the solution jet with the moderate concentration gradient can solidify (left). As the volume of the interior reduces with the evaporation of the solvent, the skin layer which was already been more or less solidified will yield to the inside, resulting in the irregular cross section (right). 


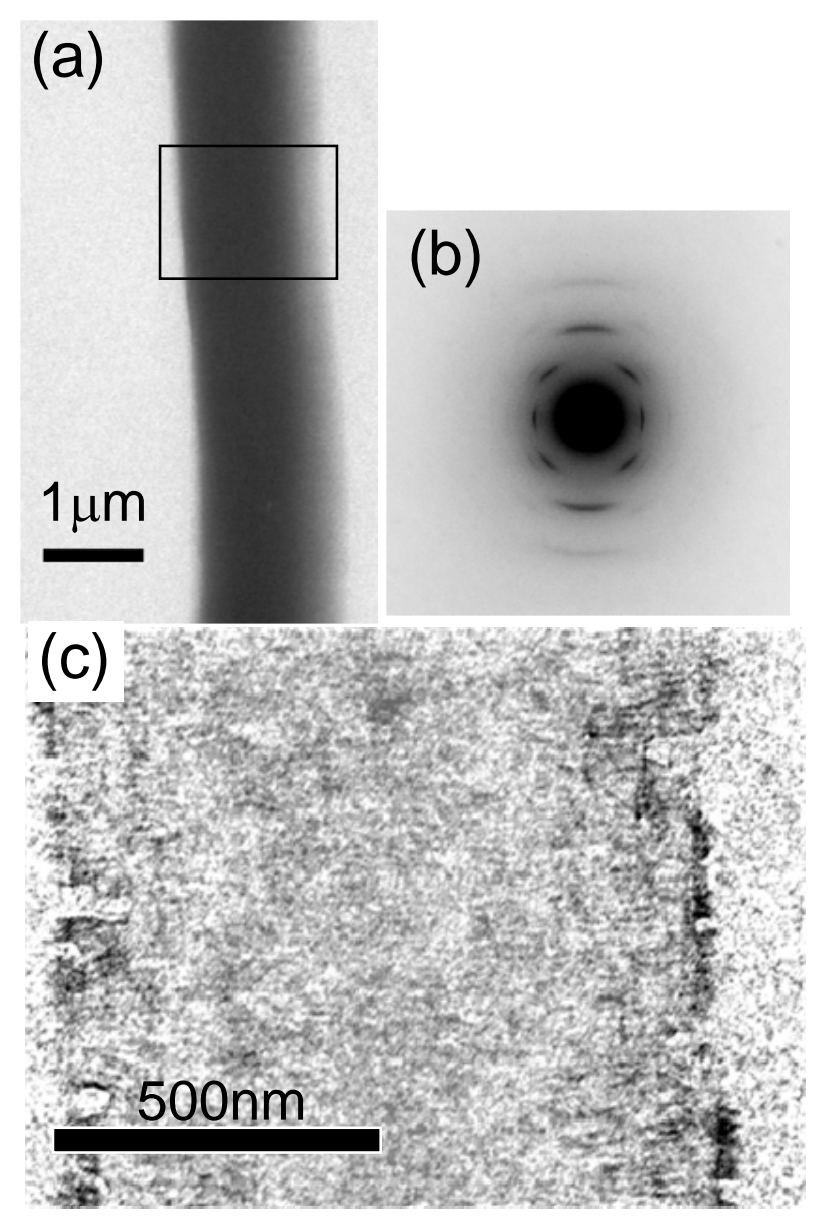

Fig. 7 Annealed ultrafine fiber of i-PS electrospun from the $1.0 \mathrm{wt} \%$ THF solution. The voltage for electrospinning was $20 \mathrm{kV}$. Temperature and the length of annealing were $180{ }^{\circ} \mathrm{C}$ and $18 \mathrm{~h}$. The surface is relatively smooth (a). Strong reflections of oriented crystals appear in the SAED pattern (b). In the enlarged and background-subtracted view of part a indicated by the rectangle, the structure consisting of densely stacked lamellae is recognized (c). 


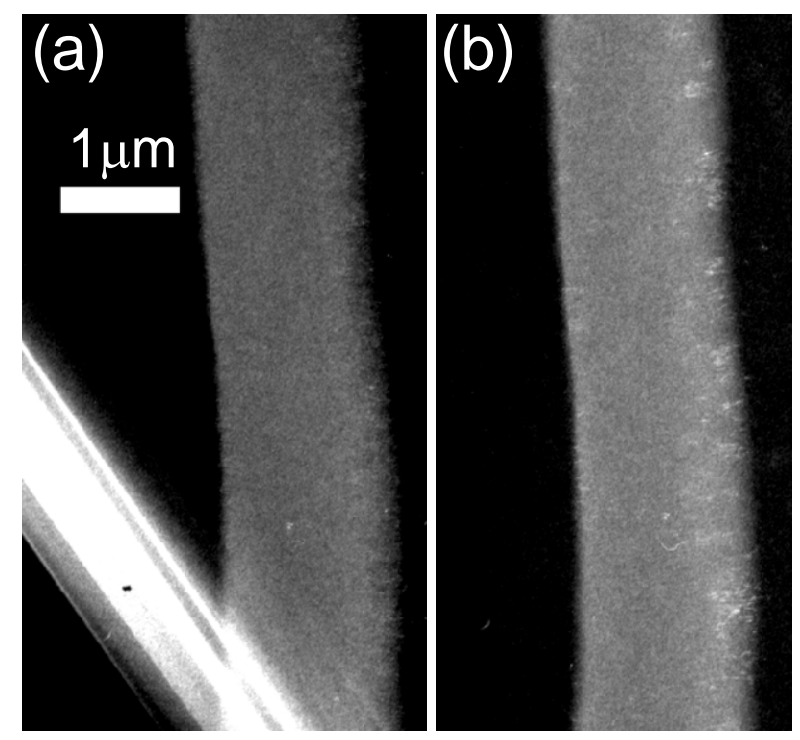

Fig. 8 DF images of the annealed i-PS fiber in Fig. 7. Reflections for the DF imaging were 102 (a) and 220 (b). In part b, observed crystallites are localized on the both sides of the fiber. 


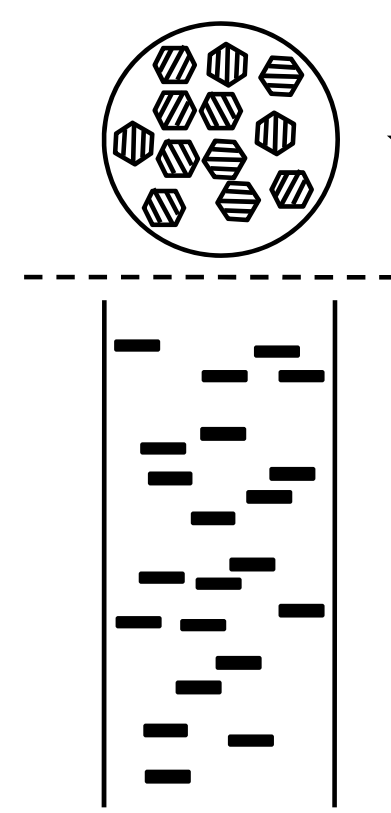

(a)
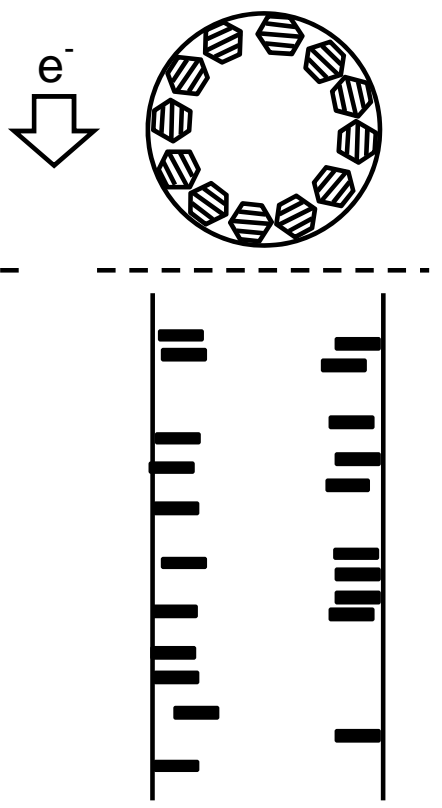

(b)

Fig. 9 Relationship between the distribution of the crystals and corresponding DF images. Upper part illustrates the cross section of the fiber. Small hexagons are the lamellar crystals with the stripes indicating the orientation of the (220) planes. The arrow in the center represents the direction of incident electron beam. Lower part illustrates the corresponding DF images with vertically aligned fiber-axis. The small rectangles represent the crystallites that satisfy the diffraction condition of the 220 reflections, in which the (220) planes are almost parallel to the incident electron beam. If the crystallites are randomly distributed in the fiber with the statistical orientation of (220) plane around the fiber axis, more crystallites will be observed near the center of the fiber (a). When the crystallites are located near the surface and the (220) planes are oriented almost parallel to the fiber surface, the crystallites are observed near the both sides of the fiber (b). 